\title{
Picropodophyllin suppresses the proliferation and invasion of hepatocellular carcinoma under serum starvation
}

\author{
MINORU TOMIZAWA and OSAMU YOKOSUKA \\ Department of Medicine and Clinical Oncology, Graduate School of Medicine, Chiba University, Chiba 260-8670, Japan
}

Received May 16, 2008; Accepted July 9, 2008

DOI: $10.3892 / \mathrm{mmr} 00000013$

\begin{abstract}
Hepatocellular carcinoma (HCC) cells invade interstitial connective tissue and proliferate. Suppression of this invasion and proliferation could serve as a novel molecular therapy. Since insulin-like growth factor-I receptor (IGF-IR) interacts with components of interstitial tissue, we focused on the inhibition of IGF-IR, analyzing HLE and HLF, poorlydifferentiated HCC cells, as well as PLC/PRF/5 and Huh-7, well-differentiated HCC cells. Cell numbers were counted under serum starvation. Western blot analysis showed that all cell lines clearly expressed IGF-IR. Either a neutralizing antibody of IGF-IR or picropodophyllin (PPP), a specific inhibitor of IGF-IR, were used, with MTS assay, to analyze changes in cell number or, with wound assay, to analyze invasion. HLF significantly proliferated without serum while the cell numbers of the other cell lines did not. IGF-IR antibody did not affect HLF cell number, while PPP decreased it. Wound assay clearly indicated that PPP inhibited the invasion of HLF. H\&E staining showed that apoptosis caused the inhibition of proliferation and metastasis. In conclusion, IGF-IR was involved in the proliferation and invasion of HCC cells in interstitial connective tissue. It was proposed that an inhibitor of IGF-IR, PPP, would be a good candidate for molecular therapy for HCC via the suppression of proliferation/invasion in interstitial connective tissue.
\end{abstract}

\section{Introduction}

Hepatocellular carcinoma (HCC) arises in liver cirrhosis after long-term infection with hepatitis B or C virus. Since HCC nodules are surrounded by fibrous septa, during metastasis HCC tumor cells invade the interstitial tissue, which consists of collagen or elastic fiber (1). Once they have invaded, tumor cells survive the apoptotic stimuli of the microenviroment of interstitial connective tissue, which include low nutrients and

Correspondence to: Dr Minoru Tomizawa, Department of Medicine and Clinical Oncology, Graduate School of Medicine, Chiba University, 1-8-1 Inohana, Chuo-ku, Chiba City, Chiba 260-8670, Japan.

E-mail: nihminor-cib@umin.ac.jp

Key words: MTS assay, neutralizing antibody, wound assay, metastasis growth factors (2). Indeed, invading HCC cells have mitotic potential (3). The mechanism behind the proliferation and invasion of HCC cells in interstitial connective tissue remains to be determined. Serum starvation has been used as a model of the microenvironment of interstitial connective tissue (4). Under serum starvation, it was revealed that a subclone of Lewis lung carcinoma that had a lower expression level of caspase-3 than its parent was resistant to apoptosis (5).

Insulin-like growth factor-I receptor (IGF-IR) is involved in cancer proliferation and invasion (6). IGF-IR mediates the proliferation and invasion signal of IGF-I and IGF-II (6), and protects cancer cells against microenvironmental stress, such as hypoxia, low $\mathrm{pH}$ or low glucose (7). Since these microenvironmental stresses are characteristic of interstitial connective tissue, it could be speculated that IGF-IR plays a role in the proliferation and invasion of HCC cells in such areas. In transgenic mice with IGF-IR, invasiveness and metastasis are promoted via integrin $(8,9), \alpha_{v} \beta_{3}$ integrin modulates IGF-IR phosphorylation by altering the rate of recruitment of Src homology 2-containing phosphotyrosine phosphatase to the activated IGF-IR. IGF-IR interacts with decorin, a proteoglycan in interstitial connective tissue that is involved in cell proliferation and survival via Akt and P21 (10). Based on the above, it is reasonable to suppose that the inhibition of IGF-IR would suppress the cell proliferation and invasion of HCC cells in interstitial connective tissue.

Antisense, neutralizing antibody, dominant negative mutant and specific inhibitors of IGF-IR inhibited the proliferation and metastasis of cancer cells when stimulated by IGF-I (11-14). However, no reports exist on the suppression of proliferation and metastasis by the inhibition of IGF-IR in a serum-free state along with IGF-I, mimicking the microenvironment of interstitial connective tissue.

Picropodophyllin (PPP), a specific inhibitor of IGF-IR, inhibits the tyrosine phosphorylation of Y1136 in the activation loop of the IGF-IR kinase domain, and successfully inhibits cell proliferation $(15,16)$.

In the search for a molecular therapy for HCC, we addressed the possibility that PPP could suppress the proliferation and invasion of HCC cells under serum starvation conditions. Our focus was on the suppression of interstitial invasion.

\section{Materials and methods}

Culture and counting of cell numbers. HLE, HLF, PLC/PRF/5 and Huh-7, all hepatocellular carcinoma cell lines, were purchased from Riken Cell Bank (Tsukuba, Japan) and cultured 
in Dulbecco's Minimum Essential Medium (DMEM) (Sigma, St. Louis, MO) supplemented with $10 \%$ fetal bovine serum (FBS) (Trace Scientific, Melbourne, Australia) in 5\% carbon dioxide at $37^{\circ} \mathrm{C}$ in a humidified chamber. When PPP (Wako Pure Chemicals, Osaka, Japan) or monoclonal anti-human IGF-IR antibody (R\&D Systems, Minneapolis, MN) were used, cells split and were cultured for $24 \mathrm{~h}$ with $10 \%$ FBS. Twenty-four hours after $10^{4}$ cells were spread onto each well of 6-well plates (Asahi Techno Glass, Tokyo, Japan), the medium was changed to a serum-free one. Cell numbers were counted after spreading on days 1,4 , and 7 by the trypan blue dye exclusion test.

Western blot analysis. After the cells had been cultured for $72 \mathrm{~h}$ in serum-free medium, $20 \mu \mathrm{g}$ protein was isolated from them, subjected to sodium dodecyl sulphate polyacylamide gel electrophoresis, then transferred to a nylon filter. Primary antibodies were polyclonal rabbit anti-IGF-IR antibody (Cell Signaling Technology, Danvers, MA) and mouse monoclonal anti-Tubulin- $\alpha$ antibody (Lab Vision, Fremont, CA). Secondary antibodies were horseradish peroxidase (HRP)-linked antirabbit antibody (Amersham Bioscience, Tokyo, Japan) and HRP-linked anti-mouse antibody (Amersham Bioscience). Dilutions were 1:500 for primary antibodies and 1:1000 for secondary antibodies. The filter was reprobed with antiTubulin- $\alpha$ antibody. Specific antigen-antibody complexes were visualized by enhanced chemiluminescence (GE Healthcare Bio-Sciences Corp., Piscataway, NJ).

Analysis of IGF-I and IGF-II secretion. Media were harvested after $72 \mathrm{~h}$ of culture without FBS. Concentrations of unbound IGF-I and IGF-II in the cell culture were measured by a 2-step enzymatic sandwich immunoassay with the Human IGF-I Immunoassay (R\&D Systems) and the Active IGF-II-ELISA (Diagnostic System Laboratories, Sinsheim, Germany), respectively.

Cell proliferation assay. Cells were trypsinized, harvested and spread onto 96-well flat-bottom plates (Asahi Techno Glass) at a density of 1000 cells/well. Following $24 \mathrm{~h}$ of culture under DMEM with $10 \%$ FBS, the medium was changed to DMEM without FBS to quench the FBS effects. After $24 \mathrm{~h}$ of culture under DMEM without FBS, IGF-II (Wako Pure Chemicals) was added to the medium. After 72 h, 3-(4,5-dimethylthiazol2-yl)-5-(3-carboxymethoxyphenyl)-2-(4-sulfophenyl)-2Htetrazolium inner salt (MTS) assay was performed according to the manufacturer's instructions (Promega Corporation, Tokyo, Japan). MTS was bio-reduced by cells into a colored formazan product that reduces absorbance at $490 \mathrm{~nm}$. Absorbance was analyzed with a multiple plate reader at a wavelength of $490 \mathrm{~nm}$ with a Bio-Rad Model 550 microplate reader (Bio-Rad, Hercules, CA).

Wound assay. Wound assays were performed according to a reported procedure (17). Briefly, cells were spread onto 4-well chambers (Beckton Dickinson, Franklin Lakes, NJ) then cut with a sterile razor. Subsequently, five images were taken with the IMT-2, a light microscope (Olympus, Tokyo, Japan). For each experiment, the number of HLF cells that had migrated $>150 \mu \mathrm{m} / 100 \mu \mathrm{m}$ of cut surface was counted.

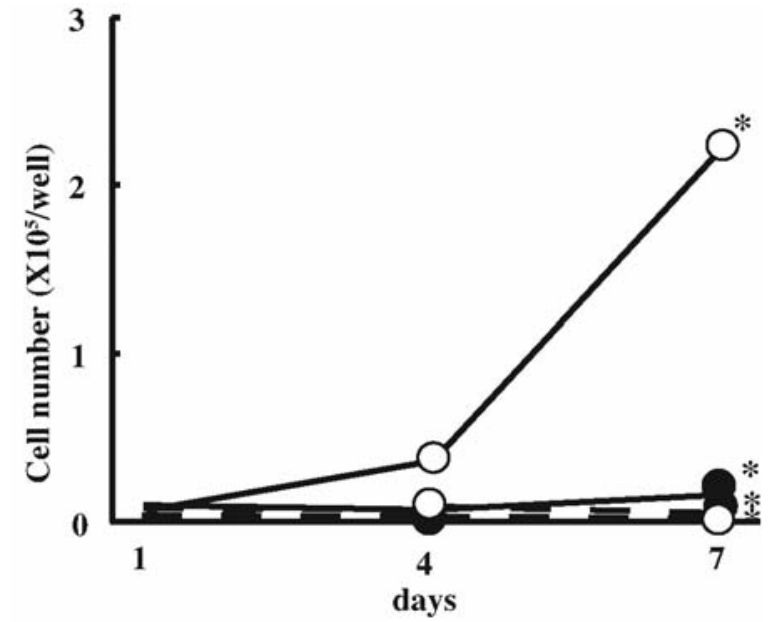

Figure 1. Change in cell number in serum-free medium. Cell numbers were counted under serum starvation. HLF significantly proliferated while the other cells did not. HLE, closed circles with solid line; HLF, open circles with solid line; PLC, closed circles with dotted line; Huh-7, open circles with dotted line. ${ }^{*} \mathrm{P}<0.05$.

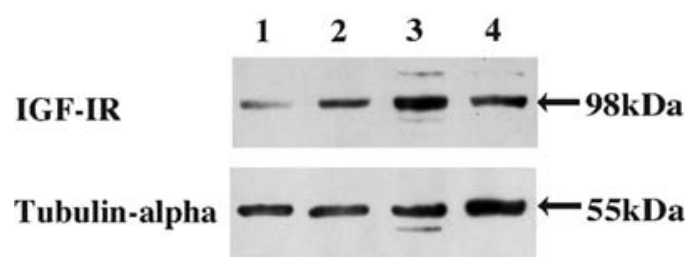

Figure 2. Western blot analysis of IGF-IR. Western blot analysis was performed with cells under serum starvation. All the cell lines expressed IGF-IR. Lane 1, HLE; 2, HLF; 3, PLC/PRF/5; 4, Huh-7.

Statistical analysis. Cell proliferation, demonstrated by MTS assay, was analyzed statistically by one-factor analysis of variance. Statistical analysis was performed with JMP5.0J (SAS Institute Japan, Tokyo, Japan). $\mathrm{P}<0.05$ was accepted as statistically significant.

\section{Results}

To determine whether any HCC cell lines had proliferated in the serum-free medium, we analyzed the cell numbers of two poorly-differentiated (HEL and HLF) and two welldifferentiated (PLC and Huh-7) HCC cell lines. HLF had significantly proliferated to $2.3 \times 10^{5}$ cells/well on day 7 , while the other cell lines did not exhibit proliferation (Fig. 1).

Western blot analysis was performed to clarify whether IGF-IR was expressed under serum starvation conditions. All the cell lines clearly expressed IGF-IR, suggesting that IGF-IR might play a role in cell proliferation (Fig. 2).

Concentrations of IGF-I and IGF-II were measured to reveal whether there was an autocrine mechanism of the IGF axis. In all cell lines, IGF-I and IGF-II were not produced or released into the medium.

We addressed the possibility that the inhibition of IGF-IR would suppress cell proliferation. MTS assay was performed after the addition of anti-IGF-IR antibody or of PPP. PPP significantly suppressed the proliferation of HLF to $18 \pm 5 \%$ 

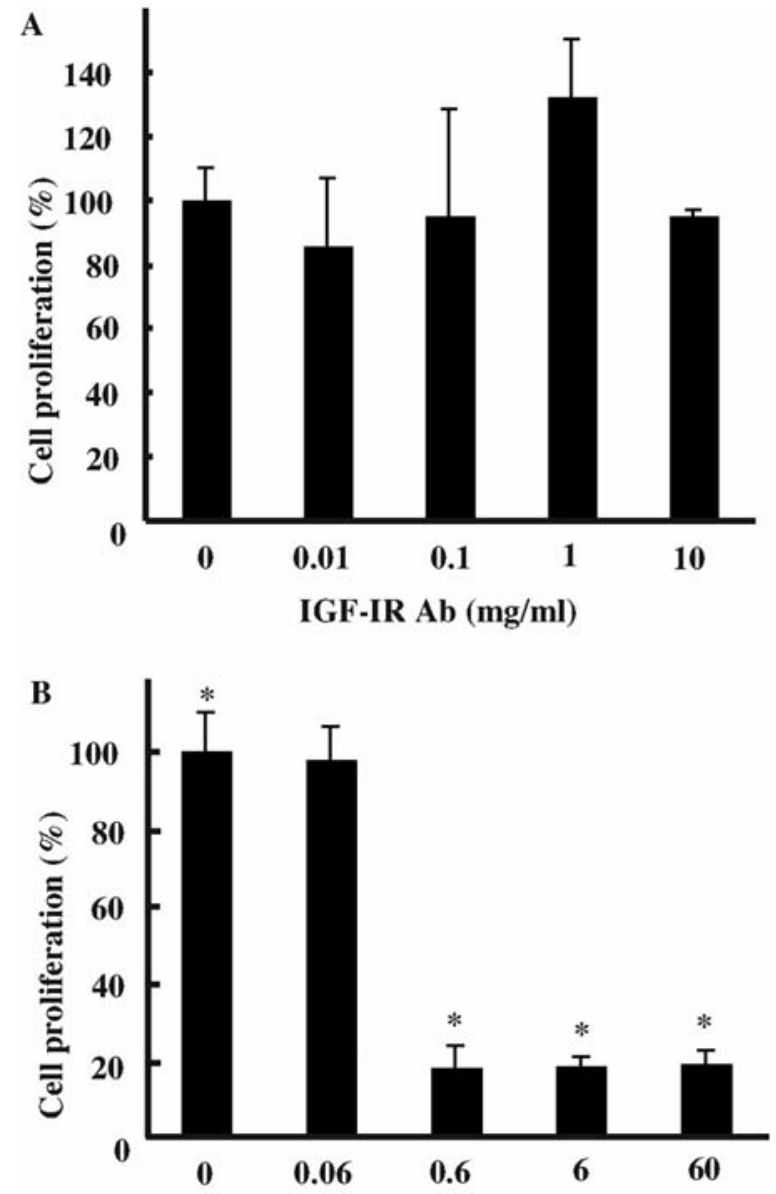

Picropodophyllin (mM)

Figure 3. Cell proliferation suppressed with picropodophyllin but not IGF-IR antibody. MTS assay was performed to analyze the suppression of cell proliferation with anti-IGF-IR antibody or pircopodophyllin. Anti-IGF-IR antibody did not suppress (A), while PPP significantly suppressed (B) the proliferation of HLF. ${ }^{*} \mathrm{P}<0.05$.

(mean \pm standard deviation), $18 \pm 3 \%$ and $19 \pm 4 \%$ at $0.6,6$ and $60 \mu \mathrm{M}$, respectively (Fig. 3A). On the other hand, anti-IGF-IR antibody did not cause cell numbers to change significantly (Fig. 3B).

In addition, changes in motility brought about by PPP were analyzed by wound assay (Fig. 4A). PPP significantly suppressed the motility of HLF, as no cells migrated $>150 \mu \mathrm{m}$ (Fig. 4B). H\&E staining was performed to observe morphological changes between HLF with and without PPP. Cells with PPP had pyknotic nuclei, while none without PPP did (Fig. 4C).

\section{Discussion}

HLF cells proliferated under serum starvation while HLE, PLC and Huh-7 cells did not. These HLF cells were cloned from the cancer tissue of a 68-year-old male patient with undifferentiated hepatocellular carcinoma with spindle-shaped cells (18). HLF cells have one allele deleted and a missense mutation in the other allele of the p53 gene, with an amino acid substitution at the 244 residue from glycine to alanine (19). They exhibit no morphological changes under either $10 \%$ FCS condition or serum starvation, but their proliferation has not been analyzed

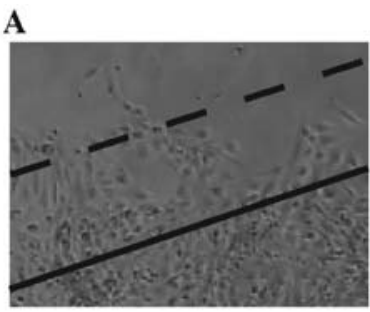

$\mathbf{0}$

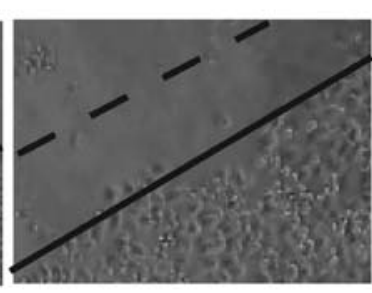

60
Picropophyllin (mM)

B
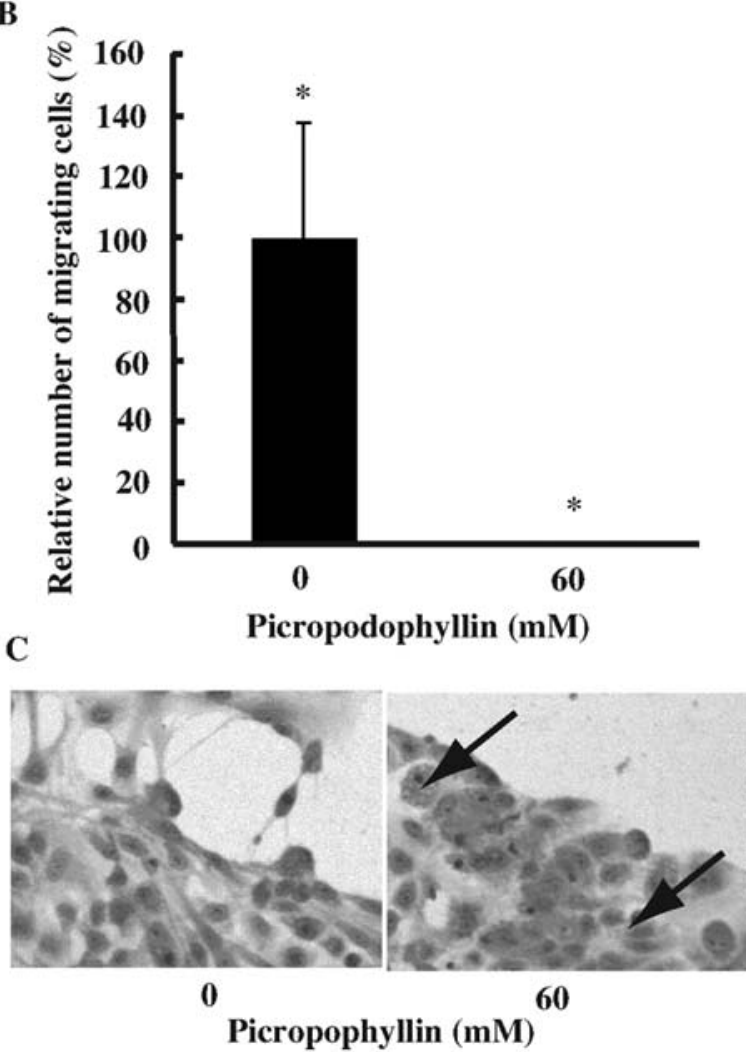

Figure 4. Cell motility was suppressed with picropodophyllin. Wound assay was performed to analyze cell motility (A). No cells cultured with PPP migrated $>150 \mu \mathrm{m}$, indicating that PPP significantly suppressed cell motility (B). H\&E staining clearly showed that PPP caused apoptosis in HLF with pyknotic nuclei $(\mathrm{C}){ }^{*} \mathrm{P}<0.05$, arrows: pyknotic nuclei.

(19). Our results clearly indicate that HLF cells proliferate under serum starvation conditions. Genetic alteration, such as that of $\mathrm{p} 53$, might provide proliferative potential under serum starvation conditions. Interestingly, $\mathrm{HCC}$ cells are spindle shaped, like HLF cells, when they invade interstitial connective tissue in thin tumor cell cords (3). HLF cells are therefore a suitable model for the analysis of the proliferation and invasion of HCC cells in interstitial connective tissue.

IGF-I and IGF-II, deeply involved in proliferation and differentiation, bind to IGF-IR (6). Upon ligand binding, tyrosines of the intracellular portion of the $B$-subunit are autophosphorylated. IGF-IR mediates their stimuli through phosphatidyl-inositol 3 kinase or mitogen-activated protein kinase (6). In the present study, all the cell lines expressed IGF-IR under serum starvation, suggesting that IGF-IR might play a role in cell proliferation or survival. This hypothesis is supported by the fact that under serum starvation proliferation 
is suppressed by a dominant negative mutant and an antisense of IGF-IR $(12,20)$. Our data show that IGF-I and IGF-II did not exist under serum starvation conditions, and that neutralizing antibody did not suppress cell proliferation. We speculated that IGF-IR was activated without stimuli under serum starvation. Tyrosines of IGF-IR are weakly phosphorylated under serum starvation, which is suggestive of its activation (21). PPP inhibits the tyrosine phosphorylation of Y1136, specific to IGF-IR (15). Thus, under serum starvation, PPP as expected inhibited cell proliferation through the inhibition of IGF-IR. Moreover, as PPP suppressed the cell motility of HLF cells under serum starvation, the speculation that PPP suppresses the invasion of HCC cells into interstitial connective tissue might well prove to be true. Indeed, this is supported by a study reporting that the antisense of IGF-IR suppresses the invasion of rat prostate cancer, inoculated under the skin of nude mice, into the brain (11).

It can be concluded that, in terms of its ability to suppress interstitial invasion, IGF-IR is a promising candidate for molecular therapy for HCC. Our follow-up study will focus on the analysis of the signaling pathways and the mechanisms of activation of IGF-IR in HLF under serum starvation.

\section{Acknowledgements}

This study was supported by a Research Grant-in-Aid for Scientific Research from the Japan Society for the Promotion of Science.

\section{References}

1. Kondo Y, Kondo F, Wada K and Okabayashi A: Pathologic features of small hepatocellular carcinoma. Acta Pathol Jpn 36: 1149-1161, 1986.

2. Gupta GP and Massague J: Cancer metastasis: building a framework. Cell 127: 679-695, 2006.

3. Tomizawa M, Kondo F and Kondo Y: Growth patterns and interstitial invasion of small hepatocellular carcinoma. Pathol Int 45: 352-358, 1995.

4. Boraldi F, Annovi G, Paolinelli-Devincenzi C, Tiozzo R and Quaglino D: The effect of serum withdrawal on the protein profile of quiescent human dermal fibroblasts in primary cell culture. Proteomics 8: 66-82, 2007.

5. Takasu M, Tada Y, Wang JO, Tagawa M and Takenaga K: Resistance to apoptosis induced by microenvironmental stresses is correlated with metastatic potential in Lewis lung carcinoma. Clin Exp Metastasis 17: 409-416, 1999.
6. Samani AA, Yakar S, LeRoith D and Brodt P: The role of the IGF system in cancer growth and metastasis: overview and recent insights. Endocr Rev 28: 20-47, 2007.

7. Peretz S, Kim C, Rockwell S, Baserga R and Glazer PM: IGF1 receptor expression protects against microenvironmental stress found in the solid tumor. Radiat Res 158: 174-180, 2002.

8. Lopez T and Hanahan D: Elevated levels of IGF-1 receptor convey invasive and metastatic capability in a mouse model of pancreatic islet tumorigenesis. Cancer Cell 1: 339-353, 2002.

9. Shen MR, Hsu YM, Hsu KF, Chen YF, Tang MJ and Chou CY: Insulin-like growth factor 1 is a potent stimulator of cervical cancer cell invasiveness and proliferation that is modulated by alphavbeta3 integrin signaling. Carcinogenesis 27: 962-971, 2006.

10. Schonherr E, Sunderkotter C, Iozzo RV and Schaefer L: Decorin, a novel player in the insulin-like growth factor system. J Biol Chem 280: 15767-15772, 2005.

11. Burfeind P, Chernicky CL, Rininsland F, Ilan J and Ilan J: Antisense RNA to the type I insulin-like growth factor receptor suppresses tumor growth and prevents invasion by rat prostate cancer cells in vivo. Proc Natl Acad Sci USA 93: 7263-7268, 1996.

12. Dunn SE, Ehrlich M, Sharp NJ, et al: A dominant negative mutant of the insulin-like growth factor-I receptor inhibits the adhesion, invasion, and metastasis of breast cancer. Cancer Res 58: 3353-3361, 1998

13. Hofmann F and Garcia-Echeverria C: Blocking the insulin-like growth factor-I receptor as a strategy for targeting cancer. Drug Discov Today 10: 1041-1047, 2005.

14. Hopfner M, Huether A, Sutter AP, Baradari V, Schuppan D and Scherubl H: Blockade of IGF-1 receptor tyrosine kinase has antineoplastic effects in hepatocellular carcinoma cells. Biochem Pharmacol 71: 1435-1448, 2006.

15. Girnita A, Girnita L, Del Prete F, Bartolazzi A, Larsson O and Axelson M: Cyclolignans as inhibitors of the insulin-like growth factor-1 receptor and malignant cell growth. Cancer Res 64: 236-242, 2004.

16. Tomizawa $\mathrm{M}$ and Saisho H: Signaling pathway of insulin-like growth factor-II as a target of molecular therapy for hepatoblastoma. World J Gastroenterol 12: 6531-6535, 2006.

17. Pennisi PA, Barr V, Nunez NP, Stannard B and Le Roith D: Reduced expression of insulin-like growth factor I receptors in MCF-7 breast cancer cells leads to a more metastatic phenotype. Cancer Res 62: 6529-6537, 2002.

18. Dor I, Namba M and Sato J: Establishment and some biological characteristics of human hepatoma cell lines. Gann 66: 385-392, 1975.

19. Terai S, Noma T, Kimura T, Nakazawa A, Kurokawa F and Okita K: Wild-type p53 gene-induced morphological changes and growth suppression in hepatoma cells. J Gastroenterol 32: 330-337, 1997

20. Adachi Y, Lee CT, Coffee K, et al: Effects of genetic blockade of the insulin-like growth factor receptor in human colon cancer cell lines. Gastroenterology 123: 1191-1204, 2002.

21. Vasilcanu D, Girnita A, Girnita L, Vasilcanu R, Axelson M and Larsson O: The cyclolignan PPP induces activation loop-specific inhibition of tyrosine phosphorylation of the insulin-like growth factor-1 receptor. Link to the phosphatidyl inositol-3 kinase/Akt apoptotic pathway. Oncogene 23: 7854-7862, 2004. 